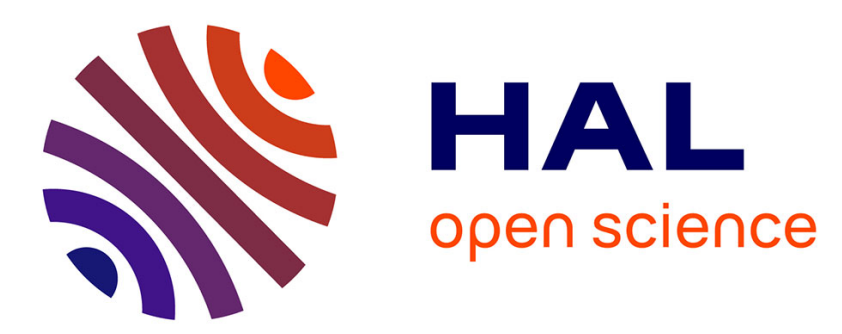

\title{
Efficient Kernel Computation for Volterra Filter Structure Evaluation
}

José Henrique de Morais Goulart, Phillip Mark Seymour Burt

\section{To cite this version:}

José Henrique de Morais Goulart, Phillip Mark Seymour Burt. Efficient Kernel Computation for Volterra Filter Structure Evaluation. IEEE Signal Processing Letters, 2012, 19 (3), pp.135 - 138. 10.1109/LSP.2011.2182342 . hal-01871272

\section{HAL Id: hal-01871272 \\ https://hal.science/hal-01871272}

Submitted on 10 Sep 2018

HAL is a multi-disciplinary open access archive for the deposit and dissemination of scientific research documents, whether they are published or not. The documents may come from teaching and research institutions in France or abroad, or from public or private research centers.
L'archive ouverte pluridisciplinaire $\mathbf{H A L}$, est destinée au dépôt et à la diffusion de documents scientifiques de niveau recherche, publiés ou non, émanant des établissements d'enseignement et de recherche français ou étrangers, des laboratoires publics ou privés. 


\title{
Efficient kernel computation for Volterra filter structure evaluation
}

\author{
J. H. Goulart and Phillip M. S. Burt
}

\begin{abstract}
Despite their generality, conventional Volterra filters are inadequate for some applications, due to the huge number of parameters that may be needed for accurate modelling. When a state-space model of the target system is known, this can be assessed by computing its kernels, which also provides valuable information for choosing an adequate alternate Volterra filter structure, if necessary, and is useful for validating parameter estimation procedures. In this paper, we derive expressions for the kernels by using the Carleman bilinearization method, for which an efficient algorithm is given. Simulation results are presented, which confirm the usefulness of the proposed approach.
\end{abstract}

Index Terms-Nonlinear systems, Volterra filters.

\section{INTRODUCTION}

V OLTERRA filters (VF) are discrete-time models used in several digital signal processing applications to represent nonlinear systems [1], [2], [3]. They provide an input/output relation that is characterized by a set of points of hypersurfaces called Volterra kernels.

For systems that admit a Volterra representation and have dynamic nonlinearities, the series underlying the VF is composed by an infinite number of kernels. In [4] it was shown that, if the system has fading memory, then its associated operator can be approximated within arbitrary precision by a Volterra model with a finite number of kernels. This number depends on the desired precision, on system characteristics and on the set of bounded inputs considered (see [4, Ths. 1, 3]).

The major drawback of a conventional VF (CVF) is the exponential growth of its number of parameters with the order and the memory of the model, which causes severe problems particularly when the modelled system is strongly nonlinear. This has motivated several researchers to study alternate Volterra filter (AVF) structures that offer a good compromise between precision and structural complexity [2], [3], especially when real time use (and adaptation) is sought.

In some applications, an approximate physical model (PM) of the target system may be available, in the form of statespace equations [5]. However, it may be the case that only typical parameter values are known, and not the exact ones. Furthermore, the model may represent a class of devices whose parameters vary, as occurs, e.g., in a production line. In this situation, the use of a VF is often more convenient, due to the simplicity in the formulation of the parameter estimation

The authors are with the Lab. of Comm. and Signals, Depart. of Telec. and Control Eng. of EPUSP, University of São Paulo, Brazil (e-mail: jgoulart@lcs.poli.usp.br; phillip@lcs.poli.usp.br).

Copyright (c) 2010 IEEE. Personal use of this material is permitted. However, permission to use this material for any other purposes must be obtained from the IEEE by sending a request to pubs-permissions@ieee.org. or adaptation problem [1], since the model is linear in the parameters. Still another advantage of a VF is its guaranteed stability that stems from the absence of feedback, which is not true for PMs.

However, the use of an AVF structure as those in [2], [3] may be necessary in order to avoid excessive computational complexity. In principle, this can be assessed with the calculation of the kernels from the state equations, by which one can estimate how many parameters a CVF should have to meet a certain precision level. If needed, an adequate AVF structure can be chosen with the information given by those kernels, which are also useful as a reference for further evaluation of the $\mathrm{AVF}$ and even for validating parameter estimation procedures, possibilities that seem unexplored in the literature (in general, the kernels used in simulations are either artificial, and thus unrealistic, or obtained from measured signals, and thus affected by noise and estimation uncertainties).

Although several methods have been proposed for calculating kernels related to state equations [6], [7], some are not appropriate for practical use. In this paper, the problems involved in the use of the approaches of [6] will be briefly described. After that, we will develop expressions for the kernels of a CVF, given a PM, by using the Carleman bilinearization method [7], [8], Also, we propose an efficient implementation for this method, which has shown itself to be effective for the problem at hand. Finally, important aspects to consider when using the computed kernels for evaluating a VF structure are discussed and simulation results are presented.

\section{Problem statement}

The input/output relation of a conventional Volterra filter with triangular kernels [3] is given by

$$
\begin{aligned}
y(n)=\sum_{p=1}^{P} \sum_{k_{1}=0}^{N_{p}-1} \ldots \sum_{k_{p}=k_{p-1}}^{N_{p}-1} v_{p}\left(k_{1}, \ldots, k_{p}\right) \\
\times u\left(n-k_{1}\right) \ldots u\left(n-k_{p}\right)
\end{aligned}
$$

where $v_{p}\left(k_{1}, \ldots, k_{p}\right)$ is the $p$-th order kernel, with memory extension (in samples) $N_{p}$, and $P$ is the maximum nonlinearity degree of the CVF. To model a physical system by (1), we consider the structure shown in Fig. 1, which operates as follows. First, the excitation sequence $u(n)$ is converted to the input signal, $u(t)$, which passes through the reconstruction filter $G_{1}(j \Omega)$, resulting in $u_{c}(t)$. Following that, $u_{c}(t)$ is fed to the continuous-time system, denoted by the $V[\cdot]$ operator, which produces the response $y_{c}(t)$. This signal, in turn, excites the anti-aliasing filter $G_{2}(j \Omega)$, resulting in the output signal 


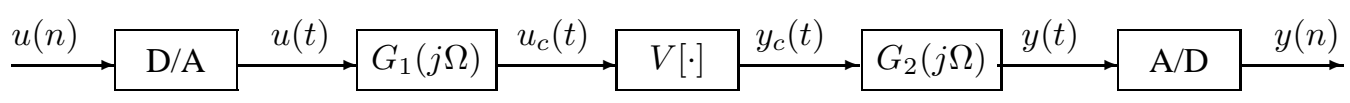

Fig. 1. Structure considered to obtain a reference conventional Volterra filter model of the physical system $V$.

$y(t)$, which is sampled to produce $y(n)$. For simplicity, we will consider that $V$ is time invariant.

From the expressions given in [7, pp. 62-63], we conclude that the continuous-time system formed by the series connection of $G_{1}, V$ and $G_{2}$ can be represented by the Volterra series

$$
\begin{aligned}
y(t)=\sum_{p=1}^{\infty} \int_{0}^{\infty} \ldots \int_{\tau_{p-1}}^{\infty} \tilde{h}_{p}\left(\tau_{1}, \ldots, \tau_{p}\right) \\
\quad \times u\left(t-\tau_{1}\right) \ldots u\left(t-\tau_{p}\right) d \tau_{p} \ldots d \tau_{1}
\end{aligned}
$$

with kernels $\tilde{h}_{p}\left(\tau_{1}, \ldots, \tau_{p}\right)$ such that their generalized frequency response functions (GFRFs)

$$
\begin{aligned}
\tilde{H}_{p}\left(j \Omega_{1}, \ldots, j \Omega_{p}\right)=\int_{0}^{\infty} & \ldots \int_{0}^{\infty} \tilde{h}_{p}\left(\tau_{1}, \ldots, \tau_{p}\right) \\
& \times e^{-j\left(\Omega_{1} \tau_{1}+\ldots+\Omega_{p} \tau_{p}\right)} d \tau_{1} \ldots d \tau_{p}
\end{aligned}
$$

are given by

$$
\begin{array}{r}
\tilde{H}_{p}\left(j \Omega_{1}, \ldots, j \Omega_{p}\right)=\left(\prod_{q=1}^{p} G_{1}\left(j \Omega_{q}\right)\right) H_{p}\left(j \Omega_{1}, \ldots, j \Omega_{p}\right) \\
\times G_{2}\left(j \Omega_{1}+\ldots+j \Omega_{p}\right)
\end{array}
$$

where $H_{p}\left(j \Omega_{1}, \ldots, j \Omega_{p}\right)$ is the $p$-th order GFRF of $V$, which is assumed to admit a Volterra representation. Basically, this requires the existence of a series that, under certain conditions, converges and provides an exact representation of $V$ [8].

The complete structure of Fig. 1 is a discrete-time system, $V_{d}$, which is to be modelled by a VF. With the frequencydomain characterization of eq. (4), we can, by using the approach of [9], impose that the (discrete-time) GFRFs associated with the CVF be such that

$$
V_{p}\left(e^{j \omega_{1}}, \ldots, e^{j \omega_{p}}\right)=\tilde{H}_{p}\left(j \frac{\omega_{1}}{\pi} \Omega_{N}, \ldots, j \frac{\omega_{p}}{\pi} \Omega_{N}\right)
$$

for $-\pi \leq \omega_{i} \leq \pi, i=1, \ldots, p$, where $\Omega_{s}=2 \Omega_{N}$ is the sampling frequency. Assuming that $G_{2}$ eliminates any aliasing effects and that $\Omega_{s}$ is sufficiently large to represent the modelled system characteristics, then (5) will provide a discrete-time model analogous to $V$ on the band of interest.

From the above discussion we see that, in order to determine the GFRFs of the CVF and, consequently, its kernels, it is necessary to know $G_{1}, G_{2}$ and the GFRFs of $V$. It should be noted that this frequency-domain derivation is advantageous over the direct sampling of the time-domain kernels, because it prevents the aliasing of components with frequency greater than $\Omega_{N}$ [9].

It is also important to point out that environmental factors, as, e.g., a room acoustic response, are not included in $G_{2}$, which is simply an anti-aliasing filter but is sufficient for obtaining a reference VF model of the nonlinear system alone. For applications where such factors are present, it can be shown from (5), eq. 23 in [7, p. 62] and a discrete-time version of it that a linear system $G_{E}$ between $V$ and $G_{2}$ can be perfectly taken into account by a discrete-time linear system after $V_{d}$, which remains unaffected. ${ }^{1}$ Thus, in the following, we will assume that $G_{1}$ and $G_{2}$ are fixed and known, focusing ourselves on the determination of the GFRFs $H_{p}\left(j \Omega_{1}, \ldots, j \Omega_{p}\right)$ from the state equations of the PM of $V$.

\section{KERNELS COMPUTATION IN THE FREQUENCY-DOMAIN}

Let us consider that an approximate PM of $V$ is known, consisting in state equations in the linear-analytic form [7], [8]

$$
\mathbf{x}^{\prime}(t)=\mathbf{f}(\mathbf{x}(t))+\mathbf{g}(\mathbf{x}(t)) u_{c}(t) \quad y_{c}(t)=\lambda(\mathbf{x}(t))
$$

where $\mathbf{x} \in \mathbb{R}^{m}, \mathbf{x}^{\prime}$ is its derivative w.r.t. time and $\mathbf{f}, \mathbf{g}$ and $\lambda$ are analytic functions of $\mathbf{x}$. This form is motivated by the fact that it represents a wide class of real systems [8] and admits a convergent Volterra representation for a set of bounded inputs, provided there exists a solution to the unforced differential equation [7]. It should be stressed out that the analyticity of the functions in (6) is essential, basically because the Volterra series arises from their Taylor series approximation around the initial state. We will also suppose that $\lambda: \mathbb{R}^{m} \rightarrow \mathbb{R}$ is a linear function $\lambda(\mathbf{x})=\mathbf{c}^{T} \mathbf{x}$, that the initial state is $\mathbf{x}(0)=\mathbf{0}$ and the solution for $u(t) \equiv 0$ is $\mathbf{x}(t) \equiv \mathbf{0}$, simplifications that, in the present case, do not imply in loss of generality [7].

In [6], two approaches are proposed for systems in this form. The first one is through closed formulas involving the solution of the unforced differential eq. (the "flow of f"), which is obviously difficult to obtain for general systems. Also, using those formulas with numerical integration instead is not a good alternative, because they involve a time-reversed flow, making the corresponding differential eqs. unstable (assuming the system is stable). The other method consists in approximating the kernels by their Taylor series and requires the use of a symbolic computation package, since the expressions involve repeated differentiations. In practice, many terms may be necessary for a good approximation, which implies a high computational cost and makes this approach unfeasible.

A technique that has shown itself adequate in practice, for allowing an accurate and efficient computation, is known as Carleman bilinearization (or Carleman linearization [7], [8]). Additionally, it conveniently permits the direct calculation of the GFRFs, without the necessity of computing time-domain kernels. In the rest of this section, we will show how this can be done and propose an efficient algorithmic implementation.

\section{A. Carleman bilinearization}

The central idea of Carleman bilinearization consists in representing the dynamics of the nonlinear system by a differential eq. in the bilinear form, for whose kernels and GFRFs

\footnotetext{
${ }^{1}$ If, however, it is desired that the reference discrete-time model include environmental factors, then, of course, $G_{E}$ can be absorbed in $G_{2}$.
} 
there are closed formulas. The brief review given below is based on [7]. For clarity, the time argument will be omitted.

Suppose that the first $P$ kernels are desired. The starting point is to calculate the Taylor series expansions of $\mathbf{f}$ and $\mathbf{g}$, up to the $P$-th order, around the initial state. This gives

$$
\mathbf{x}^{\prime}=\sum_{i=1}^{P} \mathbf{F}_{i} \mathbf{x}^{(i)}+\sum_{i=0}^{P-1} \mathbf{G}_{i} \mathbf{x}^{(i)} u
$$

with the vector $\mathbf{x}^{(i)}$ containing every $i$-th order product of the elements of $\mathbf{x}$, according to the formula

$$
\mathbf{x}^{(i)}=\mathbf{x} \otimes \mathbf{x}^{(i-1)}
$$

where $\otimes$ is the Kronecker product, $\mathbf{x}^{(1)}=\mathbf{x}$ and $\mathbf{F}_{i}, \mathbf{G}_{i} \in$ $\mathbb{R}^{m \times m^{i}}$. Then, by using the rule

$$
\left(\mathbf{x} \otimes \mathbf{x}^{(i-1)}\right)^{\prime}=\mathbf{x}^{\prime} \otimes \mathbf{x}^{(i-1)}+\mathbf{x} \otimes\left(\mathbf{x}^{(i-1)}\right)^{\prime}
$$

the bilinear differential eq.

$$
\left(\mathbf{x}^{\otimes}\right)^{\prime}=\mathbf{F} \mathbf{x}^{\otimes}+\mathbf{G} \mathbf{x}^{\otimes} u+\mathbf{b} u
$$

is constructed from (7), where

$$
\mathbf{x}^{\otimes} \triangleq\left[\begin{array}{lll}
\left(\mathbf{x}^{(1)}\right)^{T} & \ldots & \left(\mathbf{x}^{(P)}\right)^{T}
\end{array}\right]^{T}
$$

The first $m$ rows of $\mathbf{F}, \mathbf{G}$ and $\mathbf{b}$ are obtained directly from eq. (7) and the others calculated using (8) [7, pp. 108-110].

It is evident that, for $m, i>1$, the vector $\mathbf{x}^{(i)}$ necessarily has redundant components. For practical purposes, it is more efficient to compute each row of $\mathbf{F}, \mathbf{G}$ and $\mathbf{b}$ of eq. (9) separately, disconsidering the redundancies to obtain a state vector with exactly one component for each product $x_{i_{1}} \ldots x_{i_{k}}$, with $1 \leq i_{j} \leq m$ and $k \leq P$. This will be explored by the algorithm described in the next subsection.

An important property of the Carleman method is that it gives exact kernels, provided that the expansions are calculated up to the desired nonlinearity degree. This is due to the form assumed by the matrices $\mathbf{F}$ and $\mathbf{G}$ [7].

\section{B. Efficient implementation}

The inputs are the expansions of the components of $\mathbf{f}$ and $\mathbf{g}$, and thus completely determine the first $m$ rows of $\mathbf{F}, \mathbf{G}$ and b. Note that, if a symbolic computation package is available, they can be obtained computationally. The strategy proposed is capable of efficiently computing non-redundant (and, consequently, smaller) versions of $\mathbf{F}, \mathbf{G}$ and $\mathbf{b}$ by exploring two aspects of the Carleman method. First, the elements of $\mathbf{F}, \mathbf{G}$ and $\mathbf{b}$ which belong to a same row correspond, actually, to the coefficients of the (truncated) Taylor expansion that gives the evolution of the associated component of $\mathbf{x}^{\otimes}$. Second, since each element $x_{i_{1}} \ldots x_{i_{k}}$ of $\mathbf{x}^{\otimes}$ with $1<k \leq P$ can be expressed as a product of preceding ones, we can compute its associated row recursively by using preceding row vectors analogously with the rule

$$
\begin{aligned}
\left(x_{i_{1}} \ldots x_{i_{k}}\right)^{\prime}=\left(x_{i_{q+1}} \ldots x_{i_{k}}\right) \times\left(x_{i_{1}} \ldots x_{i_{q}}\right)^{\prime} & \\
& +\left(x_{i_{1}} \ldots x_{i_{q}}\right) \times\left(x_{i_{q+1}} \ldots x_{i_{k}}\right)^{\prime}
\end{aligned}
$$

TABLE I

TOTAL NUMBER OF OPERATIONS: RATIO OF THE DIRECT TO THE PROPOSED APPROACH FOR SOME VALUES OF $m$ AND $P$.

\begin{tabular}{r|rrrr}
\hline & $m=2$ & $m=3$ & $m=4$ & $m=5$ \\
\hline$P=2$ & 1.14 & 1.50 & 1.78 & 2.00 \\
$P=3$ & 2.69 & 5.30 & 8.22 & 11.20 \\
$P=4$ & 6.87 & 21.56 & 45.36 & 76.60 \\
$P=5$ & 18.47 & 97.13 & 286.02 & 611.83 \\
\hline
\end{tabular}

where $q=\lfloor k / 2\rfloor$ and $1 \leq i_{j} \leq m$. In other words, the computation of each row of eq. (9) corresponds to a symbolic derivation of the expansion it represents in terms of two previously derived expansions, which are represented by preceding rows. Note that the (symbolic) multiplications of eq. (11) can be implemented efficiently by shifting the elements of the vectors according to the ordering of (the non-redundant) $\mathbf{x}^{\otimes}$, and the sum is performed as a usual vector sum.

A MATLAB version of this implementation was made available in [10]. It is significantly more efficient than the direct computation described in [7, pp. 108-110]. However, due to space limitations, we will not derive this gain, which is a complicated function of $m$ and $P$ [10] and is due to the fact that the redundancy eliminated with our algorithm grows exponentially with these variables. For instance, for $m=3$ and $P=4$, the non-redundant $\mathbf{F}$ and $\mathbf{G}$ contain less than $9 \%$ of the elements of their redundant counterparts. To exemplify the improvement, in Table I the ratio of the number of operations required by the direct approach to the number required by the proposed one is given for some values of $m$ and $P$.

\section{Calculating the kernels with use of the GFRFs}

With $\mathbf{F}, \mathbf{G}$ and $\mathbf{b}$ and defining $\mathbf{c}_{V} \triangleq\left[\begin{array}{llll}\mathbf{c}^{T} & 0 & \ldots & 0\end{array}\right]^{T} \in \mathbb{R}^{M_{P}}$, being $M_{P}$ the dimension of $\mathbf{b}$, the GFRFs of the system (9) can be calculated by [7]

$$
H_{p}\left(j \Omega_{1}, \ldots, j \Omega_{p}\right)=\mathbf{c}_{V}^{T} \mathbf{W}_{p} \mathbf{G} \ldots \mathbf{G} \mathbf{W}_{2} \mathbf{G} \mathbf{W}_{1} \mathbf{b}
$$

with $p=1, \ldots, P$, where

$$
\mathbf{W}_{k}=\left(j\left(\Omega_{1}+\ldots+\Omega_{k}\right) \mathbf{I}_{M_{P}}-\mathbf{F}\right)^{-1}, \quad k=1, \ldots, p
$$

In principle, with the GFRFs $H_{p}$ and the filters $G_{1}$ and $G_{2}$, the kernels of the CVF can be determined through the inverse transform of (5). In practice, assuming that the $p$-th kernel contains little energy after some instant $T_{p}$, an approximation of the anti-transforms of (5) that will suffice is [9]

$$
v_{p}\left(n_{1}, \ldots, n_{p}\right)=\operatorname{IFFT}\left\{\tilde{V}_{p}\left(k_{1}, \ldots, k_{p}\right)\right\}
$$

with $0 \leq k_{1}, \ldots, k_{p}<N_{p}$, where

$$
\tilde{V}_{p}\left(k_{1}, \ldots, k_{p}\right)=\tilde{H}_{p}\left(j \Omega_{s} \frac{2 k_{1}-N_{p}}{2 N_{p}}, \ldots, j \Omega_{s} \frac{2 k_{p}-N_{p}}{2 N_{p}}\right)
$$

and $N_{p}=\left\lceil T_{p} \Omega_{s} / 2 \pi\right\rceil$. As observed in [9], it is important to note that this corresponds to sampling a circular convolution of the kernels with the sinc function in the time-domain, and thus there will be a non-causal portion of the kernels appearing at samples next to $N_{p}$, which may impair the results. One way of attenuating this effect is to multiply the right hand side of eq. (15) by a multidimensional sinusoidal window. 


\section{VOLTERRA FILTER STRUCTURE EVALUATION}

The kernels associated with the PM provide many valuable information for choosing an appropriate VF structure, as explained next. First, by using a set of inputs with variances increasing from about zero up to the desired maximum $\sigma_{\max }^{2}$, we can determine which combination of filter order $P$ and memories $N_{p}, p=1, \ldots, P$, leads to a sufficiently accurate CVF. This is done by comparing the responses of some CVFs with different $P$ and $N_{p}$ to those inputs with the respective responses produced by the system of Fig. 1, which are computed by means of numerical integration of the PM (and filtering). To quantify this comparison, a useful measure is the fit index

$$
\operatorname{FIT}(\hat{\mathbf{y}})=100 \times\left(1-\frac{\|\mathbf{y}-\hat{\mathbf{y}}\|}{\left\|\mathbf{y}-\mu_{\mathbf{y}}\right\|}\right)
$$

where $\mathbf{y}$ and $\hat{\mathbf{y}}$ are vectors with samples of the outputs of the PM and the CVF, respectively, and $\mu_{\mathrm{y}}$ is the sample mean of $y$. Obviously, one should choose the CVF with the lowest resulting number of coefficients that meets the desired precision, because it is the least computationally costly to use.

Second, if this complexity is inevitably high, some characteristics of the computed kernels may be explored for choosing an adequate AVF structure. For instance, if the kernels are smooth, then a sparse-interpolated scheme, as proposed in [2], can be used. Also, if the energy of the kernels is concentrated around their main diagonals, a diagonal representation with limited width can be used, as suggested in [3].

\section{Simulation Results}

To exemplify the discussed evaluation, the kernels of first to fourth orders associated with the loudspeaker PM of [5] were computed. Since it involves a non-analytic function $L(x)$, it was first adjusted by replacing this curve by an approximation in the form $L_{a}(x)=l_{0}+l_{1} \operatorname{tg}^{-1}\left(\omega_{l} x+\phi_{l}\right)$. The sampling rate chosen was $F_{s}=5 \mathrm{kHz}$. For the memory, it was verified that $N=100$ is a good upper bound to $N_{p}, p=1, \ldots, 4$, hence the kernels were calculated initially with this memory. Then, two CVFs were defined: $H_{V_{1}}$, with the four kernels and $N_{1}=\ldots=N_{4}=100$, and $H_{V_{2}}$, with only the three first kernels and $N_{1}=100, N_{2}=N_{3}=90$. The inputs used were 50 different realizations of a Gaussian noise sequence with 500 samples having zero mean and with energy only up to approximately $625 \mathrm{~Hz}$, since the PM represents a bass loudspeaker. Their variances were varied, and the fit indexes of the $P$-th order approximations given by $H_{V_{1}}$ and $H_{V_{2}}$ w.r.t. the output of the PM were computed for different values of $P$. The ensemble average of the results is shown in Fig. 2 as a function of the variance of the input. Immediately, we see that the linear approximations (which are identical) degrade rapidly, while the higher order ones give fairly good results. Also, we note that $H_{V_{2}}$ performs almost as good as $H_{V_{1}}$ for $P=2$ or $P=3$. Thus, if, e. g., a $98 \%$ fit is desired and $\sigma_{\max }^{2} \leq 7$ (as defined in Sec. IV), we can define the reference CVF with $P=2, N_{1}=100$ and $N_{2}=90$, resulting in 4195 coefficients. However, if $\sigma_{\max }^{2}=9$, then a sufficient choice is $P=3, N_{1}=100$ and $N_{2}=N_{3}=90$, resulting in 129775 coefficients, which is an increase by a factor of about 31 .

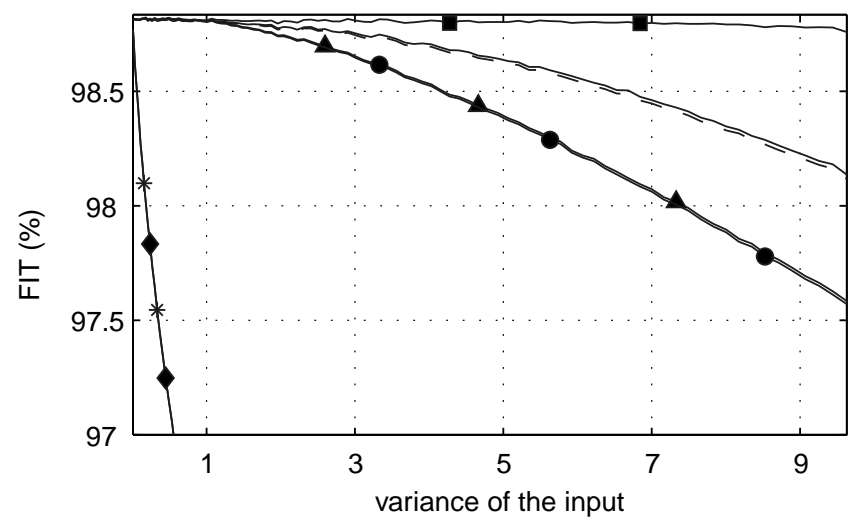

Fig. 2. Fit index of the approximations given by $H_{V_{1}}$ with orders $P=1$ $(-*), P=2(-\boldsymbol{\Delta}), P=3(-), P=4(-\mathbf{\square})$ and by $H_{V_{2}}$ with orders $P=1(-\bullet), P=2(-\bullet), P=3(--)$.

\section{CONCLUSION}

In this paper, we approached the problem of evaluating Volterra filter structures for nonlinear system modelling in DSP applications. The proposed strategy consists in computing the resulting discrete-time system kernels through expressions that involve the filters of the $\mathrm{A} / \mathrm{D}$ and $\mathrm{D} / \mathrm{A}$ converters and the physical system kernels, calculated with the Carleman bilinearization. To this end, it is necessary that a state-space model is known a priori. As was shown by simulation results, this provides valuable information for choosing an adequate VF structure, i. e., one that exhibits a satisfactory accuracy and a sufficiently low computational complexity. In addition, the computed kernels may be used as a reference for validation of parameter estimation procedures and, if an alternate Volterra filter structure is necessary, for its quantitative evaluation.

\section{REFERENCES}

[1] L. Azpicueta-Ruiz, M. Zeller, A. Figueiras-Vidal, J. Arenas-Garcia, and W. Kellermann, "Adaptive Combination of Volterra Kernels and its Application to Nonlinear Acoustic Echo Cancellation," IEEE Trans. Audio Speech Lang. Process., vol. 19, no. 1, pp. 97-110, Jan. 2011.

[2] E. Batista, O. Tobias, and R. Seara, "A sparse-interpolated scheme for implementing adaptive volterra filters," IEEE Trans. Signal Process., vol. 58, no. 4, pp. 2022-2035, Apr. 2010.

[3] M. Zeller and W. Kellermann, "Fast and Robust Adaptation of DFTDomain Volterra Filters in Diagonal Coordinates Using Iterated Coefficient Updates," IEEE Trans. Signal Process., vol. 58, no. 3, pp. 15891604, Mar. 2010.

[4] S. Boyd and L. Chua, "Fading memory and the problem of approximating nonlinear operators with Volterra series," IEEE Trans. Circuits Syst., vol. CAS-32, no. 11, pp. 1150-1161, Nov. 1985.

[5] D. Franken, K. Meerkotter, and J. Wassmuth, "Observer-based feedback linearization of dynamic loudspeakers with Ac amplifiers," IEEE Trans. Speech Audio Process., vol. 13, no. 2, pp. 233-242, Mar. 2005.

[6] A. Isidori, Nonlinear Control Systems, 3rd ed. New York: Springer, 1995.

[7] W. J. Rugh, Nonlinear System Theory: The Volterra/Wiener Approach. Baltimore, MD: Johns Hopkins University Press, 1981.

[8] R. Brockett, "Volterra series and geometric control theory," Automatica, vol. 12, no. 2, pp. 167-176, Mar. 1976.

[9] H. Koeppl and D. Schwingshackl, "Comparison of discrete-time approximations for continuous-time nonlinear systems," in Proc. IEEE Int. Conf. Acoust., Speech, Signal Process. (ICASSP), Montreal, Canada, May 2004, pp. 881-884.

[10] J. H. Goulart and P. M. S. Burt. (2011, Dec.) Code for the efficient bilinearization algorithm and complexity evaluation. [Online] Available: http://www.lcs.poli.usp.br/ jgoulart/research.html. 